\title{
Study on the Effect of Canceling Hard Bed on Patients after Renal Needle Biopsy
}

\author{
Xiaomin Zou*, Yingzi Huang*, Fanna Liu, Yu Chen\#, Yingxue Zhong\# \\ Department of Nephrology, The First Affiliated Hospital of Jinan University, Guangzhou, China \\ Email: "272171384@qq.com
}

How to cite this paper: Zou, X.M., Huang, Y.Z., Liu, F.N., Chen, Y. and Zhong, Y.X. (2021) Study on the Effect of Canceling Hard Bed on Patients after Renal Needle Biopsy. Open Journal of Urology, 11, 177-186. https://doi.org/10.4236/oju.2021.115016

Received: March 18, 2021

Accepted: May 28, 2021

Published: May 31, 2021

Copyright (c) 2021 by author(s) and Scientific Research Publishing Inc. This work is licensed under the Creative Commons Attribution International License (CC BY 4.0).

http://creativecommons.org/licenses/by/4.0/ (c) (i) Open Access

\begin{abstract}
Objective: To investigate the effect of hard bed on postoperative complications and comfort level of patients after renal puncture biopsy. Methods: A total of 115 patients who underwent renal needle biopsy in the Department of Nephrology of The First Affiliated Hospital of Jinan University from May 2020 to February 2021 were selected, and 57 patients who received hard bed nursing after renal needle biopsy were set as the control group, the others were selected as the observation group. The incidence of postoperative bleeding at the puncture mouth, gross hematuria and dysuria, as well as the postoperative comfort level of $6 \mathrm{~h}$ and $18 \mathrm{~h}$ were compared between the two groups. Results: There was no significant difference in the incidence of postoperative bleeding, gross hematuria and dysuria between the two groups $(P>$ $0.05)$, but the comfort scores at $6 \mathrm{~h}$ and $18 \mathrm{~h}$ in the observation group were higher than those in the control group $(P<0.05)$. Conclusion: There is no significant difference in the incidence of postoperative complications, such as bleeding at the puncture mouth, gross hematuria and dysuria, among patients with renal needle biopsy, whether to use hard bed nursing or not. Eliminate hard bed nursing can improve the comfort level of patients, reduce the occurrence of pressure ulcers, and relieve the negative psychological emotions of patients.
\end{abstract}

\section{Keywords}

Hard Bed, Renal Puncture Biopsy, Postoperative Complications

\section{Introduction}

In recent years, chronic kidney disease has become one of the serious public health problems worldwide [1]. Renal needle biopsy is one of the effective ex-

${ }^{\star}$ Co-first author.

\#Corresponding author. 
amination means for the diagnosis of a variety of renal diseases, and can guide the treatment and determine the prognosis. However, as an invasive operation, postoperative complications such as puncture bleeding, infection, gross hematuria, and dysuria are prone to occur [2] [3] [4]. In order to reduce postoperative bleeding and other complications, traditional postoperative nursing requires patients to rest on a hard bed for $24 \mathrm{~h}$ [5] [6]. Long time immobilization on a hard bed will cause pain in the patient's back, and make the patients uncomfortable [7]. With the improvement of renal puncture technology, the nursing mode to postoperative patients has also changed. Present, several literatures have reported that nursing after renal needle biopsy no longer strictly requires immobilization on a hard bed, and the immobilization time and position have also been adjusted [8] [9] [10]. Previous studies have conducted a series of beneficial explorations on the time and body position of immobilization, but the effects of not using hard bed nursing are not reported yet [11]. This paper aims to explore the effects of not using hard bed nursing on postoperative complications and comfort of patients with renal needle biopsy through a comparative study.

\section{Materials and Methods}

\subsection{General Information}

The subjects of this study were patients who underwent renal needle biopsy in the First Affiliated Hospital of Jinan University from May 2020 to February 2021. Inclusion criteria: patients without contraindications to renal biopsy, who were clearly diagnosed by renal biopsy, clearly aware, willing to cooperate with the questionnaire, and signed the informed consent. Exclusion criteria: severe coagulation dysfunction or bleeding tendency, kidney tumor patients, or patients with severe ascites or hydronephrosis, mental abnormalities, and heart failure.

According to the order of admission, 57 patients with renal needle biopsy from May 2020 to September 2020 were set as the control group, and 58 patients from October 2020 to February 2021 were set as the observation group. The difference between the two groups was that no hard bed was used in the observation group. There are 27 males and 30 females in the control group. Their age composition is as follows: 30 patients are younger than 40 years old, 24 cases are between 40 and 65 years old, and 3 cases are older than 65 years old. Education degree: 9 cases are primary school or below, 24 cases are middle school or technical secondary school, 24 cases were junior college or above. The length of hospitalization of patients ranged from 4 to 40 days, with an average of 14.8 days. The prothrombin time of patients ranged from 11.8 to 15.2 seconds, with an average of 13.06 seconds, and the thrombin time ranged from 14.8 to 22.5 seconds, with an average of 17.98 seconds. The observation group has 29 males and 29 females. Their age composition is as follows: 20 cases are younger than 40 years old, 32 cases are between 40 and 65 years old, and 6 cases are older than 65 years old. Education degree: 20 cases are primary school or below, 19 cases are middle 
school or technical secondary school, 19 cases were junior college or above. The length of hospitalization ranged from 4 to 54 days, with an average of 13.5 days. The prothrombin time of patients ranged from 11.8 to 16.0 seconds, with an average of 13.22 seconds, and the thrombin time ranged from 13.0 to 21.0 seconds, with an average of 17.55 seconds. There is no significant difference in general data between the two groups (Table 1, Table 2).

\subsection{Methods}

\subsubsection{Preoperative Preparation}

Preoperatively instruct patients to practice defecation and urination on the bed, inhale and close breath, instruct patients not to eat blood-activating food such as Angelica sinensis and Codonopsis pilosula, and nursing fully check whether patients are using anticoagulant drugs, and remind doctors to postpone the puncture if there is.

\subsubsection{Intraoperative Nursing}

During the operation, assist patients in the prone position, with a hard pillow on the lower abdomen, conventional disinfection and towel laying, local infiltration anesthesia with $2 \%$ lidocaine, one-time biopsy needles were used to puncture the

Table 1. Comparison of general data of patients (\%).

\begin{tabular}{|c|c|c|c|c|}
\hline Items & $\begin{array}{l}\text { Control group } \\
\qquad(\mathrm{n}=57)\end{array}$ & $\begin{array}{l}\text { Observation group } \\
\qquad(\mathrm{n}=58)\end{array}$ & $x^{2}$ & $P$ \\
\hline Gender & & & 0.080 & 0.778 \\
\hline male & $27(47.4)$ & $29(50)$ & & \\
\hline female & $30(52.6)$ & $29(50)$ & & \\
\hline \multicolumn{5}{|l|}{ Age/year } \\
\hline$<40$ & $30(52.6)$ & $20(34.5)$ & 4.134 & 0.127 \\
\hline $40-65$ & $24(42.1)$ & $32(55.2)$ & & \\
\hline$>65$ & $3(5.3)$ & $6(10.3)$ & & \\
\hline Education degree & & & 5.327 & 0.070 \\
\hline Primary School or Below & $9(15.8)$ & $20(34.5)$ & & \\
\hline $\begin{array}{l}\text { Middle school or technical } \\
\text { secondary school }\end{array}$ & $24(42.1)$ & $19(32.8)$ & & \\
\hline College degree or above & $24(42.1)$ & $19(32.8)$ & & \\
\hline
\end{tabular}

Table 2. Comparison of general data of patients (mean $\pm \mathrm{SD})$.

\begin{tabular}{ccccc}
\hline Group & Number & Hospitalization days & $\begin{array}{c}\text { Prothrombin time } \\
\text { (seconds) }\end{array}$ & $\begin{array}{c}\text { Thrombin time } \\
\text { (seconds) }\end{array}$ \\
\hline Control group & 57 & $14.80 \pm 8.23$ & $13.06 \pm 0.68$ & $17.98 \pm 1.73$ \\
Observation group & 58 & $13.55 \pm 8.13$ & $13.22 \pm 0.72$ & $17.55 \pm 1.65$ \\
$t$ & & 0.823 & 1.18 & 1.372 \\
$P$ & 0.412 & 0.241 & 0.173 \\
\hline
\end{tabular}


lower pole cortex of the kidney twice successively under the supervision of color ultrasound, and two renal tissues were taken for pathology.

\subsubsection{Postoperative Nursing}

Assist the patient to shift over the bed, and instruct the patient to lie in bed for 24 hours. During the first 6 hours, the patient was absolutely supine, and the patient was instructed to perform upper limb exercises and ankle pump exercises to prevent deep vein thrombosis, and the patient was told to avoid lumbar exertion. The patients were given ECG monitoring and blood oxygen saturation monitoring for $6 \mathrm{~h}$. At the same time, the patients were told to drink more water appropriately and not to eat blood-promoting food. The control group was given absolute supine on hard bed for 6 hours after operation.

The postoperative urine color of the patients was observed ( 1 cup was kept for each of the first 3 urinations for comparative observation). Abnormal conditions such as bleeding from dressing and lumbago were observed in each shift for three consecutive days. Keep nursing records, and report to the doctor in time if the above cases occur. Observe the bleeding situation of the puncture mouth of the patient $6 \mathrm{~h}$ and $18 \mathrm{~h}$ after surgery, and give the patient a General Comfort Questionnaire [12].

\subsection{Observation and Evaluation Criteria}

\subsubsection{Postoperative Gross Hematuria, Extravasation of Blood from Puncture Mouth and Dysuria}

After the patients were sent back to the ward after surgery, they were given 3 white urine cups and urine was collected for 3 consecutive times. The patients were observed and recorded whether there was gross hematuria or not. The bleeding situation of the puncture dressing was observed for three days after operation, and detailed records were made. The occurrence of dysuria was determined by whether the patient had indwelling catheter after surgery.

\subsubsection{Comfort Level at $6 \mathrm{~h}$ and $18 \mathrm{~h}$ after Surgery}

The postoperative comfort of patients was assessed by using the simplified comfort status scale developed by American comfort nursing expert Kolcaba. In order not to affect patients' rest, a comfort questionnaire was conducted at $6 \mathrm{~h}$ and $18 \mathrm{~h}$ after the puncture. The scale contains a total of four dimensions of physiology, psychology, spirit, social culture and environment, with a total of twenty-eight items, including five items in the physiological dimension, ten items in the mental and spiritual dimension, six items in the social dimension, and seven items in the environmental dimension., Using Likert four-level scoring method, one point means very disagree, four points means very agree, the higher the score, the more comfortable.

\subsection{Statistical Methods}

The SPSS21.0 statistical software was used to conduct statistical processing on the data. Count data were represented as cases and percentages, and chi-square 
test was adopted. Measurement data was expressed by mean values plus standard deviation, and $\mathrm{T}$-test was adopted. The difference was statistically significant with $P<0.05$.

\section{Results}

\subsection{Comparison of Postoperative Gross Hematuria, Puncture Port Bleeding and Dysuria in the Two Groups}

After renal biopsy, the control group and the observation group did not show statistical differences in postoperative complications such as gross hematuria, bleeding from the puncture port and dysuria $(P>0.05)$ (Table 3$)$.

\subsection{Comparison of Comfort Scores between the Two Groups of Patients at $6 \mathrm{~h}$ and $18 \mathrm{~h}$ after Surgery}

The comfort scores of the observation group at $6 \mathrm{~h}$ and $18 \mathrm{~h}$ after surgery were significantly higher than those of the control group, and the difference was statistically significant $(P<0.01)$ (Table 4$)$.

\section{Discussion}

Renal needle biopsy is one of the most important clinical means for the diagnosis, treatment and prognosis judgment of kidney diseases. However, as an invasive operation, it is often accompanied by various postoperative complications, postoperative nursing if it is not in place, will increase the suffering of patients and affect the prognosis. With the increasingly mature and widespread development of renal puncture technology, how to reduce and alleviate postoperative complications and discomfort of patients has become a concern of clinical nursing.

In order to reduce the occurrence of complications such as bleeding, the early

Table 3. Comparison of postoperative gross hematuria, puncture port bleeding and dysuria between the two groups (\%).

\begin{tabular}{ccccc}
\hline Group & Number & Gross hematuria & Puncture port bleeding & Dysuria \\
\hline Control group & 57 & $7(12.3)$ & $5(8.8)$ & $6(10.5)$ \\
Observation group & 58 & $4(6.9)$ & $4(6.9)$ & $8(13.8)$ \\
$\chi^{2}$ & & 0.963 & 0.14 & 0.287 \\
$P$ & 0.326 & 0.978 & 0.592
\end{tabular}

Table 4. Comparison of postoperative comfort scores at $6 \mathrm{~h}$ and $18 \mathrm{~h}$ between the two groups (mean $\pm \mathrm{SD}$ ).

\begin{tabular}{cccc}
\hline Group & Number & $6 \mathrm{~h}$ & $18 \mathrm{~h}$ \\
\hline Control group & 57 & $73.23 \pm 3.82$ & $74.44 \pm 4.46$ \\
Observation group & 58 & $80.60 \pm 3.06$ & $80.81 \pm 4.35$ \\
$t$ & & 11.424 & 7.752 \\
$P$ & & $<0.01$ & $<0.01$ \\
\hline
\end{tabular}


nursing after renal biopsy usually binds bellyband and requires the patient to lie supine on a hard bed for 6 hours and absolutely rest in bed for 24 hours, which brings discomfort to the patient. This nursing process is a summary of predecessors' clinical medical nursing experience and its actual nursing effect lacks relevant evidence-based basis [13] [14]. At present, there have been reports of renal biopsy in foreign countries that patients do not need too much activity restriction and professional nursing after surgery [15] [16]. Therefore, many domestic studies have carried out new explorations on the traditional postoperative nursing methods of binding abdominal band and immobilization in bed for 24 hours after operation. Bao et al. [17] and Mo et al. [4] believe that the removal of abdominal band binding has little effect on the occurrence of complications after renal biopsy, but it can improve the comfort of patients. The study by Zhao et al. [18] confirmed that the bed rest time for renal puncture was shortened from the conventional $24 \mathrm{~h}$ to $12 \mathrm{~h}-15 \mathrm{~h}$, which would not increase complications and could relieve patients' discomfort.

At present, although the use of hard bed is no longer strictly required for postoperative nursing of renal needle biopsy, there are few literatures to confirm the effect of not using hard bed nursing after surgery.

At present, although the nursing after renal biopsy is no longer rigidly required to use a hard bed, there are few confirmed literatures on the impact of not using a hard bed nursing after the operation. Through detailed comparative study, this paper draws the following conclusions.

\subsection{The Absence of Hard Bed Nursing after Renal Needle Biopsy Will Not Increase the Postoperative Complications of the Patient}

The results of this study showed that there was no statistically significant difference between the two groups in the occurrence of postoperative gross hematuria and bleeding from the puncture port after surgery $(P>0.05)$. It shows that canceling the use of hard bed in the nursing process after renal puncture will not increase the postoperative hemorrhagic complications. The reason may be that the location of the kidney is deep, and the hard bed has limited effect on the compression and hemostasis. Even if it is cancelled, it will not cause obvious bleeding at the puncture port.

For patients who are bedridden for a long time, their physiological needs for urination are affected, and some patients may have dysuria, but the occurrence of dysuria is mainly related to a variety of factors such as bedridden position, environment, pain and tension [19]. In this study, there was no statistical difference in the incidence of dysuria between the two groups, indicating that the absence of a hard bed nursing will not affect dysuria in patients.

\subsection{The Postoperative Comfort of Patients Is Improved by Not Using Hard Bed Nursing}

The long-term use of hard bed after surgery will lead to patients' back discom- 
fort, pressure sores, and increase the discomfort of patients. The discomfort further causes patients' fear of renal puncture, affects postoperative recovery and increases the difficulty of clinical nursing. Therefore, nurses need to pay attention to the patient's condition in clinical nursing, and maximize the patient's comfort experience when the condition permits. The results of this study showed that the comfort level questionnaire scores of the observation group at 6 $\mathrm{h}$ and $18 \mathrm{~h}$ after surgery were significantly higher than those of the control group, and the differences were statistically significant $(P<0.01)$, indicating that the not using a hard bed can improve the comfort of patients after renal needle biopsy.

\section{Conclusion}

In summary, the non-use of hard bed in routine nursing after renal needle biopsy has no significant effect on postoperative complications such as bleeding at the puncture port, gross hematuria, dysuria, etc., and can improve the postoperative comfort of patients, which provides a reference for whether to use hard bed nursing after renal biopsy. However, the sample size of this study is small. In the follow-up study, the sample size will be increased and more possible influencing factors will be included for further research.

\section{Conflicts of Interest}

The authors declare no conflicts of interest regarding the publication of this paper.

\section{References}

[1] Wang, J., Xie, X.D., Huang, J., Liu, C.B., Liu, S.S., Sun, F. and Yue, P. (2015) Qualitative Study of the Inner Experience of Nephropathy Patients Underwent Renal Puncture. Nursing Journal of Chinese People's Liberation Army, 32, 20-23.

[2] Li, Y.Z. and Wu, Q. (2021) Effect of Meticulous Nursing Intervention on Postoperative Comfort and Complications of Renal Aspiration Biopsy Patients. Journal of Clinical Medical Research and Practice, 6, 176-178.

[3] Li, S.F. and Li, W.J. (2020) Analysis of Influencing Factors of Local Hematoma Formation after Renal Biopsy Guided by Ultrasound. Journal of Imaging Research and Medical Applications, 4, 25-27.

[4] Mo, Y.W., Chen, W.H. and Xu, J. (2020) Effects of Abolishing Abdominal Band Application on Safety and Comfort of Patients after Renal Needle Biopsy. Journal of Nurses Training, 35, 1503-1505.

[5] Hu, Z.H. (2002) Nursing Experience of Patients Undergoing Renal Biopsy. Acta Medicinae Sinica, 15, 600-601.

[6] Chen, Y.M. (2011) Clinical Nursing Care of 33 Cases of Renal Biopsy Guided by Color B Ultrasound. Journal of Qilu Nursing, 17, 91-92.

[7] Li, L.S. and Liu, Z.H. (2008) Chinese Nephrology. People's Military Medical Press, Beijing, 145

[8] Song, L.M. and Wang, L.P. (2017) Analysis of Causes of Urinary Retention after Renal Puncture and Nursing Improvement. China Medicine and Pharmacy, 7, 
166-168.

[9] Xiong, S., Li, H.Q. and Ke, C.Y. (2017) Application of Standardized Nursing in Patients Undergoing Renal Biopsy. Journal of Clinic Nursing's Practicality, 34, 38-45.

[10] Liang, X.L. (2017) Perioperative Nursing Experience of Renal Biopsy under Ultrasound Guidance. Jonrnal of Practical Medicine Techniques, 24, 117-118.

[11] Pan, L.Y. and Lv, G.L. (2012) Research Progress on Bed Time and Position of Patients after Undergoing Renal Puncture Biopsy. Chinese Nursing Research, 26, 1161-1162.

[12] Kolcaba, K. (1992) Holistic Comfort: Operationalizing the Construct as a Nurse Sensitive Outcome. Advances in Nursing Science, 15, 1-10. https://doi.org/10.1097/00012272-199209000-00003

[13] Qian, L.P. (2013) Nursing Experience of Renal Biopsy. Journal of Nurses Training, 28, 144-145.

[14] Ren, Q.F., Hu, H.Y., Xu, Y.M., Guo, B.Q., Zhang, Y.L. and Zhang, W. (2011) Nursing Care of 56 Patients Undergoing B-Type Ultrasound-Guided Percutaneous Renal Biopsy. Chinese Journal of General Practice, 9, 1151-1152.

[15] Gong, J.L., Wang, L.Y., Wu, H.X. and Fan, J.J. (2017) Application of Clustered Nursing Intervention in Patients Undergoing Renal Biopsy. Chinese General Practice Nursing, 15, 4162-4164.

[16] Su, W.Q. (2012) Nursing Progress of Complications after Renal Biopsy. Chinese Journal of Clinical Rational Drug Use, 5, 168-170.

[17] Bao,Y., Wen, Y.Q. and Zhu, Y.L.(2007) Clinical Study on Whether to Tie up the Abdominal Band after Renal Biopsy. Sichuan Medical Journal, 28, 333-334.

[18] Zhao, C., Chen, Z.R. and Yang, Z.H. (2004) The Clinical Significance of Shortening the Time in Bed after Ultrasound-Guided Automatic Renal Biopsy. Guangxi Medical Journal, 26, 500-501.

[19] Gong, D.Y. and Fan, R. (2009) Cause Analysis and Nursing Progress of Patients with Urine Retention after Renal Biopsy. Nursing Journal of Chinese People's Liberation Army, 26, 238-239. 


\section{Supplementary}

Comfort score sheet of the control group and observation group at $6 \mathrm{~h}$ and $18 \mathrm{~h}$

\begin{tabular}{|c|c|c|c|}
\hline Control group (6 h) & Observation group $(6 \mathrm{~h})$ & Control group $(18 \mathrm{~h})$ & Observation group $(18 \mathrm{~h})$ \\
\hline 69 & 83 & 73 & 85 \\
\hline 68 & 82 & 79 & 84 \\
\hline 78 & 81 & 75 & 79 \\
\hline 77 & 81 & 74 & 84 \\
\hline 76 & 83 & 73 & 83 \\
\hline 79 & 79 & 79 & 79 \\
\hline 74 & 76 & 78 & 76 \\
\hline 73 & 75 & 80 & 81 \\
\hline 77 & 83 & 74 & 82 \\
\hline 71 & 85 & 71 & 86 \\
\hline 70 & 78 & 78 & 84 \\
\hline 81 & 79 & 84 & 80 \\
\hline 80 & 82 & 75 & 77 \\
\hline 82 & 81 & 79 & 78 \\
\hline 73 & 84 & 83 & 85 \\
\hline 76 & 76 & 70 & 73 \\
\hline 75 & 80 & 78 & 84 \\
\hline 76 & 77 & 77 & 84 \\
\hline 79 & 81 & 83 & 83 \\
\hline 72 & 81 & 71 & 80 \\
\hline 73 & 83 & 74 & 82 \\
\hline 70 & 82 & 79 & 77 \\
\hline 75 & 85 & 77 & 79 \\
\hline 74 & 83 & 71 & 76 \\
\hline 71 & 76 & 73 & 76 \\
\hline 77 & 80 & 84 & 77 \\
\hline 73 & 81 & 69 & 82 \\
\hline 80 & 83 & 74 & 85 \\
\hline 76 & 82 & 70 & 87 \\
\hline 73 & 84 & 76 & 83 \\
\hline 73 & 83 & 74 & 81 \\
\hline 70 & 82 & 75 & 80 \\
\hline 68 & 81 & 77 & 85 \\
\hline 69 & 73 & 70 & 76 \\
\hline 67 & 76 & 82 & 78 \\
\hline
\end{tabular}




\section{Continued}

\begin{tabular}{|c|c|c|c|}
\hline 73 & 79 & 70 & 73 \\
\hline 76 & 75 & 69 & 78 \\
\hline 71 & 75 & 68 & 77 \\
\hline 75 & 76 & 69 & 70 \\
\hline 76 & 83 & 70 & 87 \\
\hline 73 & 81 & 71 & 78 \\
\hline 73 & 83 & 72 & 76 \\
\hline 76 & 81 & 75 & 76 \\
\hline 74 & 82 & 73 & 71 \\
\hline 71 & 81 & 74 & 75 \\
\hline 72 & 83 & 82 & 85 \\
\hline 71 & 84 & 80 & 84 \\
\hline 70 & 80 & 76 & 79 \\
\hline 73 & 76 & 73 & 85 \\
\hline 69 & 79 & 69 & 87 \\
\hline 63 & 79 & 70 & 86 \\
\hline 67 & 78 & 71 & 87 \\
\hline 69 & 81 & 73 & 83 \\
\hline 73 & 83 & 73 & 83 \\
\hline 70 & 82 & 68 & 84 \\
\hline 73 & 83 & 68 & 80 \\
\hline \multirow[t]{2}{*}{71} & 88 & 70 & 87 \\
\hline & 82 & & 85 \\
\hline
\end{tabular}

